\title{
Luminous Intensity Distribution Curve Effect on Luminaire Placement
}

Abstract. The paper discusses a way to deploy a given number of point light sources in the interior to provide required illuminance levels and uniformity of the working plane by using a genetic algorithm. Furthermore a genetic algorithm has been used to generate a proper luminous intensity distribution curve of multiple luminaires to meet the given criteria for the reference plane of the model room for predefined luminaire positions.

Keywords: genetic algorithm, luminaire, luminous intensity distribution curve.

\section{Interior Lighting}

Interior lighting systems of work places on the territory of the Czech Republic have to be designed to meet among others the Czech Technical Standard ČSN EN $12464-1$ [1] Light and lighting - Lighting of work places - Part 1: Indoor work places.

For this project an administrative model room has been chosen of dimensions $10 \mathrm{~m} \times 5 \mathrm{~m}, 4$ meters high with luminaires $3.5 \mathrm{~m}$ above the floor. This model room was chosen to serve for handwriting, writing on typewriters, reading and processing data according to reference number 5.26.2 [1]. For this instance there are several conditions that have to be met:

$\bar{E}_{m}$ Maintained Average Illuminance of 500 Ix at least

$U G R_{L}$ Unified Glare Rating of 19 at most

$U_{0}$ Lighting Uniformity of 0.6 at least

$R_{a}$ General Color Rendering Index of 80 at least

To meet the mentioned standard, average illuminance of the reference plane $75 \mathrm{~cm}$ above the floor must be greater than the defined level $\bar{E}_{m}$ at all times.

The maintenance factor $M F$, defining the depreciation of design level luminous flux over the course of operation, has not been taken into account for the chosen model room. In other words, the emitted luminous flux from the point light sources and reflectances of the surfaces will stay constant over time. As a result the mentioned Maintained Average Illuminance $\bar{E}_{M}$ will become also the design illuminance, required at the beginning of the maintenance period.

Unified Glare Rating can be calculated by the following equation [1]:

(1) $U G R_{L}=8 \cdot \log _{10}\left(\frac{0.25}{L_{B}} \sum \frac{L^{2} \omega}{p^{2}}\right)$

Where:

$L_{B}$ is the luminance of the background

$L$ is the luminance of each light source in the direction of the observer

$\omega$ is the solid angle of the light source as seen by the observer

$p$ is the Guth position index

In this project simplified point light sources have been used, meaning that they are only defined by position, rotation and a luminous intensity distribution curve. Because they have no surface area, their luminance is infinite and they have no solid angle seen from any observer, therefore the $U G R_{L}$ requirement cannot be met. Furthermore calculations of luminous flux have been made without the consideration of light spectrum making parameter $R_{a}$ needless to deal with.

\section{Photometric Value Calculation}

The illuminance $E$ of a planar surface is the areal density of luminous flux incident on the surface [2]:

(2) $E=\frac{d \Phi}{d A}$

Where:

$d \Phi$ is the luminous flux incident on a surface

$d A$ is the surface's area

Luminous intensity $I$ is the amount of luminous flux contained in a given solid angle. For a direction defined by angle $\gamma$ is luminous intensity of this angle defined as follows [2]:

(3) $\quad I_{\gamma}=\frac{d \Phi}{d \Omega}$

Where:

$d \Phi$ is the amount of luminous flux contained in the solid angle $d \Omega$

$d \Omega$ is the solid angle with its axis pointing in direction $\gamma$

The used point light sources' spatial luminous flux distribution is defined by a luminous intensity distribution curve symmetrical about the vertical axis. To obtain the illuminance of a surface according to figure 1 the following equation can be used:

(4) $E_{P_{\rho}}=\frac{I_{\gamma} \cdot \cos (\beta)}{l^{2}}$

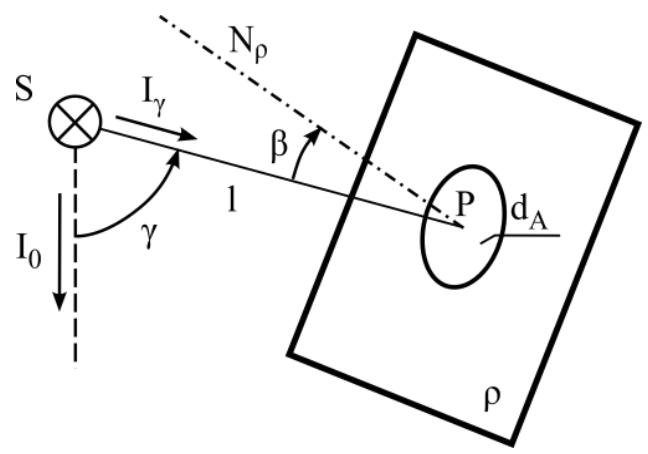

Figure 1. Facet $\rho$ illuminated by light source $S$ 
In figure 1 the normal $N_{\rho}$ and the light ray $l$ originating in light source $\mathrm{S}$ and incident on surface $d A$ form angle $\beta$ as used in (4).

To achieve more accurate luminous flux spatial distribution calculation results for the chosen indoor space, reflections from surfaces have been taken into account. Walls, ceiling and floor of the model room have been chosen to be painted with white matte interior paint. Having this in mind the simple but in this instance relatively accurate diffuse reflection model has been used for the surface reflection calculations of the model room.

If we take reflections into account, every point of the room's surfaces will become a secondary light source as seen in figure 2 .

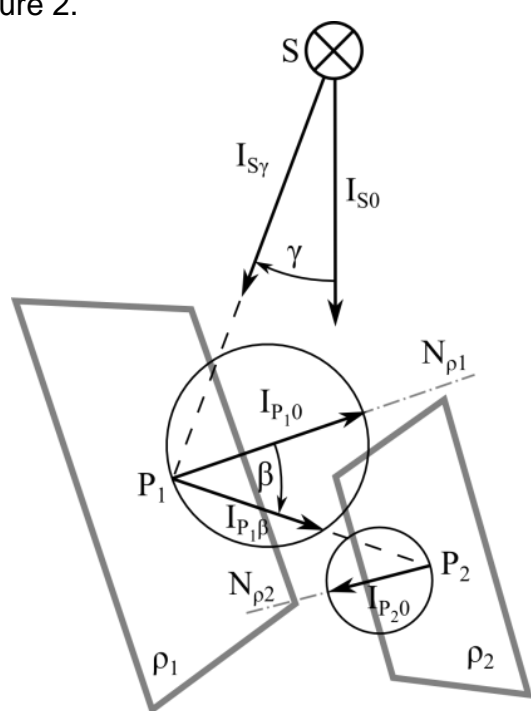

Figure 2. Multiple diffuse reflections on a light ray originating from source $S$.

For this project diffusely reflecting surfaces have been chosen showing the so called Lambertian reflectance.

According to figure 2 the primary light source $S$ will light up point $P$ of the surface $\rho_{1}$ with Lambertian reflectance and become a secondary light source, i.e. a light source with a luminous intensity distribution curve according to the following function:

(5) $I_{\beta}=I_{0} \cdot \cos (\beta)$

Where:

$I_{0}$ is the reference luminous intensity pointing in the same direction as the surface's normal

$I_{\beta}$ is the luminous intensity in direction $\beta$

$\beta$ is the angle formed between reference luminous intensity $I_{0}\left(N_{\rho}\right)$ and the required luminous intensity $I_{\beta}$, $\beta \in 0^{\circ}, 90^{\circ}$

Point $P$ can also light up another surface creating multiple reflections. Reflectance $\rho$ is the ratio of the reflected radiant or luminous flux to the incident flux in the given conditions [3]. Reflectances of the model room's surfaces are 0.8 for the ceiling, 0.5 for the walls and 0.2 for the floor meaning that 80,50 or $20 \%$ of light respectively will be reflected. Luminous flux will thus fade away with each reflection making it sufficient, for a certain degree of accuracy, to calculate only the first few reflections.

To make it possible to compute the luminous flux incident on the surfaces of the model room with reflections included, the finite element method has been used, i.e. surfaces have been divided into smaller facets. The more facets participate in luminous flux distribution calculations, the more time-consuming the calculations will become but results will be more accurate.

\section{Reflections and Radiosity}

Radiosity is a global illumination algorithm used in 3D computer graphics rendering [5]. Radiosity and the chosen model room surfaces use the same Lambertian reflectance making it possible to use Radiosity procedures within this project.

After applying the finite element method (dividing the surfaces of the model room into smaller facets), the initial luminous flux incident [4] on the facets can be calculated. All facets in visual contact with point light sources will receive a portion of their luminous flux according their distances and rotations. For the instance of the model room, all facets will receive an initial luminous flux from the used light sources.

After the initial luminous flux incidents have been calculated for each facet and reflectances $\rho$ have been applied, the facets' reflected luminous fluxes are know as well as their luminous distribution curves. From this point on, the following step can be done n-times for n-reflections:

(6) $\Phi_{F n p}=\rho_{F} \sum \Phi_{f p p \rightarrow F}$ Where:

$\Phi_{F n p}$ is the reflected luminous flux for the next pass of facet $\mathrm{F}$

$\rho_{F}$ is the reflectance of facet $\mathrm{F}$

$\Phi_{f p p \rightarrow F}$ is the luminous flux incident on facet $\mathrm{F}$ originating from facet $f$ calculated during the previous pass

After the next pass luminous fluxes have been calculated for all the facets, next pass values become previous pass values and another pass can be calculated, i.e. one reflection can be added to the final facets' luminous fluxes.

\section{Reference Plane Illuminance}

The reference plane has to be divided into facets similarly to the previous calculations. Each reference plane facet receives luminous flux directly from light sources and from visible facets from the rest of the model room as calculated in the previous chapter. To calculate average illuminance of a facet from incident luminous flux equation EQ2 can be used.

\section{Interior Lightning System Consideration}

The paper consider two task of lighting system design. The first is based on knowledge of a used lighting sources. Therefore the luminous intensity distribution curve and count of sources are known. The lightning system design only consist of determination of suitable position of the sources. This case is further called as "lighting sources positions design".

Opposite approach is considered in the second case. The known position of the sources and their count are prescribed. The algorithm tries to find suitable solution of luminous intensity curve here. This case is further called as "luminous intensity curve design"

Both task have common part of phenotype where the target average illuminance and lighting uniformity are requested. There are moreover an expectation of symmetry in case of source position design results and similar an expectation of lighting source efficiency in case of luminous curve design results. 


\section{Lighting Sources Positions Design}

The DNA was defined by several floating point values, which defined the $x$ and $y$ coordinates of each lighting source and the last one defined the multiple of luminous intensity curve. The variable multiple ensured that the target illuminance can be always reached and its limits were set between 10 and $10^{4}$. The resulting luminous intensity was get from equation:

(7) $\quad I=M \cdot f_{C}$

Where

$M$ is the searched multiple and

$f_{C}$ is normalized luminous curve function.

Table 1: DNA description for lighting source positions design

\begin{tabular}{l|l|l|l|l|l}
$\mathbf{X}_{1}$ & $\mathbf{Y}_{1}$ & $\ldots$ & $\mathbf{X}_{\mathbf{n}}$ & $\mathbf{Y}_{\mathrm{n}}$ & $\mathbf{M}$ \\
\hline
\end{tabular}

The coordinate's limits were set according to the model room dimensions $10 \mathrm{~m} \times 5 \mathrm{~m}$. The fitness function was prescribed as follows:

(8) fit $_{P}=\left(10 \cdot\left(U_{0}-U_{0 T}\right)\right)^{2}+\left(0.1 \cdot\left(\bar{E}-\bar{E}_{T}\right)\right)^{2}+c_{v}$ Where

$U_{0}, U_{0 T}$ are evaluated and target lighting uniformity,

$\bar{E}, \bar{E}_{T}$ are evaluated and target average value of illuminance and

$c_{v}$ is coefficient of variation, which ensure the symmetry of the solutions. It was defined by division of sample standard deviation and average value of illuminance.

There is strict prescription of lighting uniformity in the defined fitness function. Authors chose it by the idea that the minimal uniformity had to be reached and then the symmetry of the result is most important.

\section{Luminous Intensity Curve Design}

It was very difficult to design the DNA of the luminous intensity curve. It had to be ensured the variability of the results while there was not any negative intensity for any angle. Authors defined following function in the end:

(9) $I=M \cdot\left(\begin{array}{l}\left(B_{2} \cos ^{2} \vartheta+B_{1} \cos \vartheta\right)^{E_{B}}+ \\ +\left(A_{2} \sin ^{2} \vartheta+A_{1} \sin \vartheta\right)^{E_{A}}\end{array}\right)$

The definition of each parameter limits secured that there could not be the case of negative intensity value:

- $A_{1}, B_{1} \in\langle 1,2\rangle$

- $A_{2}, B_{2} \in\langle-1,1\rangle$

- $E_{A}, E_{B} \in\langle 1,3\rangle$

Table 2: DNA description luminous intensity curve design

\begin{tabular}{|c|c|c|c|c|c|c|}
$\mathbf{B}_{2}$ & $\mathbf{B}_{1}$ & $\mathbf{E}_{\mathrm{B}}$ & $\mathbf{A}_{2}$ & $\mathbf{A}_{1}$ & $\mathbf{E}_{\mathbf{A}}$ & $\mathbf{M}$ \\
\hline
\end{tabular}

It is obvious that the defined function restricts the set of solutions. So the results must be considered as optimum for this set and not as a global optimum.

The fitness function was prescribed as follows:

(10a) fit $_{P}=\left[\begin{array}{l}\left(10 \cdot\left(U_{0}-U_{0 T}\right)\right)^{2}+ \\ +\left(0.1 \cdot\left(\bar{E}-\bar{E}_{T}\right)\right)^{2}+0.01\end{array}\right] \cdot \Phi, \quad U_{0}<U_{0 T}$

(10b) fit $_{P}=\left\lfloor\left(0.1 \cdot\left(\bar{E}-\bar{E}_{T}\right)\right)^{2}+0.01\right] \cdot \Phi, \quad U_{0}>U_{0 T}$ Where

$U_{0}, U_{0 T}$ are evaluated and target lighting uniformity,
$\bar{E}, \bar{E}_{T}$ are evaluated and target average value of illuminance and

$\Phi$ is the total luminous flux of the source.

There is smoother rule for lighting uniformity that at least minimal uniformity must be reached. So it could be also higher in the results. The efficiency was considered by the calculated luminous flux. In case that the minimal uniformity and target average illuminance are fulfilled the minimization of the luminous flux is requsted.

\section{Settings of GA for Presented Solution}

Two ideas were studied as it was described in previous sections. The similar GA settings were used in both of them, which are described in the table 1. Explanation and some specification of the rows in the table are made in further sections. The count of generations was different for solutions of "lighting sources positions design" and "luminous intensity curve design". The solution with defined lighting curve took more time to be found. So there were set 100 generation in this case. The count of 50 generation seemed to be sufficient for the solutions with "luminous intensity curve design".

Table 3: Settings of GA
\begin{tabular}{|l|c|}
\hline First population & $\begin{array}{c}\text { Random with uniform } \\
\text { probability }\end{array}$ \\
\hline Termination condition & $\begin{array}{c}\text { Reaching the maximum of } \\
\text { generations }\end{array}$ \\
\hline Generations & $100 / 50$ \\
\hline Population size & 200 \\
\hline Recombination probability & $90 \%$ \\
\hline Mutation probability & $15 \%$ \\
\hline Parent selection mechanism & Tournament: 1 best of 4 \\
\hline Mutation mechanism & non-uniform \\
\hline Survival selection & elitism \\
\hline
\end{tabular}

\section{Parent Selection Mechanism}

There are some known possibilities how to select good solutions to the next step of the algorithm. Our early algorithm used the "roulette selection" mechanism. This type of selection is based on probability given to each member according to their fitness function. Each member could be then randomly selected to the next generation with its probability. However the prepared algorithm suffers from premature convergence. This problem is quite common in case of roulette selection. If there is an excellent member in comparison to others in the population, then it has extremely high probability to be several times selected to the next generation. The recombination process is then useless, because the whole population is created almost from the same DNA.

The searched value of luminous flux in the defined genotypes made big differences in resulting fitness functions. The value of illuminance highly depends on used (found) level of luminous flux. Other parameters in the genotype have much lower effect. Therefore even good DNAs could be removed from the population if their value of luminous flux is too high or too low. After removing the luminous flux from the genotypes, the GA algorithm works well, but we were forced to consider only the lighting uniformity.

The premature convergence was fixed by „tournament selection" eventually. The tournament selection takes randomly a small group of members. Then the defined 
count of members with highest fitness in the group continues to the next generation. Both the size of the selected group and the count of advancing members provide probability to take the low fitness members to the next generation.

The tournament selection „1 best of 4 " was used in case of our solutions.

\section{Mutation Mechanism}

The described algorithm used the non-uniform mutation with normal distribution. This type of mutation exhibits lower changes in the genotype than the uniform mutation. Also the amount of the change could be simply controlled by the standard deviation of normal distribution. We use the fact that approximately $2 / 3$ of samples lie within the interval of standard deviation.

The mutation process contain following steps:

- The DNA values were selected randomly by the probability of mutation.

- The samples from defined normal distribution was added to selected DNA values.

- The changed DNA values were cut to fit their bounds.

\section{Survival Selection}

The GA is like stochastic method of searching solutions. Note that the new generation is created from the previous one by involving members with some probability. So there is always some probability that the best solution would be replaced by the worse one during transition between two generation.

The problem can be eliminated by some survival mechanism. Authors used the elitism in the presented solution. Elitism means to take the best solution always to the next generation.

There were taken two members of the best solution in the algorithm. First one was not allowed to change its DNA, so the mutation was forbidden here. The second member was allowed to be affected by mutation, so there was let the possibility to continue of searching starting from this point.

\section{Results of the Algorithm}

The results of GA are shown in figure 3. For all solutions the minimal lighting uniformity and the target value of average illuminance were satisfied. The figures from a) to d) represents results of lighting sources position design for defined luminous intensity curve, which is shown above each solution. There can be done comparison among three types of luminous intensity curves all with six lighting sources in figures a)-c). The case a) represents very narrow curve, which is given by:

(11) $f_{C}=\cos ^{6} \vartheta$

The figure $b$ ) represents purely cosine source and figure c) include also sine part so it is the widest from all three studied curves:

(12) $f_{C}=\cos \vartheta+0.5 \cdot \sin \vartheta$

The figures from e) to $\mathrm{g}$ ) represents design of luminous intensity curve for defined positions of lighting sources, which are described in table 4 . Note that the resulting lighting uniformity can be more than the required value 0.6 . This was very important. For instance in case of solution e), the GA was not able to find smaller uniformity than 0.7 for a given description of luminous intensity curve given by equation (9).

Some test was also made to compare both design methods. The solution in figure d) was found for luminous intensity curve, which was the result for defined positions described in the fourth row of the table 4.
Table 4: Coordinates of lighting sources and resulting value of lighting uniformity and flux

\begin{tabular}{|c|c|c|c|c|}
\hline S & $x(m)$ & $y(m)$ & $\begin{array}{c}U_{0} \\
(-)\end{array}$ & $\begin{array}{c}\Phi \\
(I m)\end{array}$ \\
\hline e) & {$[1,5,9,1,5,9]$} & {$[1,1,1,4,4,4]$} & 0.76 & 12751 \\
\hline f) & {$[1,5,9,1,5,9]$} & {$[2,2,2,3,3,3]$} & 0.63 & 13240 \\
\hline g) & {$[1,9,1,9]$} & {$[1,1,4,4]$} & 0.60 & 28411 \\
\hline d) & {$[2.5,7.5,2.5,7.5]$} & {$[1,1,4,4]$} & 0.60 & 26933 \\
\hline
\end{tabular}

\section{Conclusion and Result Consideration}

As expected the wider the luminous intensity curve is the GA found the positions of lighting sources in greater distance from walls. For very narrow curves as in case of figure 3a), there appeared regular grid pattern as optimal solution. The grid pattern can be found also in case of the cosine source in figure $3 \mathrm{~b}$ ). But in this case the pattern is somehow rotated for specific angle. This rotation was probably caused by the restriction to get the exact value of lighting uniformity. The biggest distances between ligthing sources are in case of solution c). The GA always tried to set the sources in the diagonal of the room for several runs of the algorithm. It was difficult to design the positions with lighting uniformity exatly 0.6 here. So it would be suitable to make some changes in the requirements or decrease the count of sources in this solution.

As it was said above, the tests a)-d) were ended after 100 generation. It must be note that there was still good possibility that the fitness function might get lower values. However the better solution would be found by spending more time and only symmetry would get better.

The solution in figure $3 d$ ) represents the found position for luminous curve determined by another GA working with defined positions in table 4 row 4 . In both cases the exact value 0.6 of lighting uniformity was reached. Though both positions are not equal, there can be seen some similarity. The luminous curves in figures $3 d$ ) $-\mathrm{g}$ ) were found for specified positions. Solutions e) and f) consist six lighting sources and solutions d) and g) consist four lighting sources. Also from these result is sure that the wider luminous curves were found for sources with higher distance from the walls.

The GAs are not able to find exact solutions as common deterministic algorithms. Therefore several runs of the same settings gave different but similar results. This must be taken into account in case of design application.

\section{REFERENCES}

[1] ČSN EN 12464-1. Světlo a osvětlení - Osvětleni pracovních prostorů: Část 1: Vnitřní pracovní prostory. Praha: Úřad pro technickou normalizaci, metrologii a státní zkušebnoctví, 2012.

[2] HABEL, Jiří. Světlo a osvětlování. Praha: FCC Public, 2013, $622 \mathrm{~s}$. ISBN

978-80-86534-21-3.

[3] ČSN EN 12665. Light and lighting: Basic terms and criteria for specifying lighting requirements. Praha: Úřad pro technickou normalizaci, metrologii a státní zkušebnictví, 2012.
[4] TGLTLSBFSSP: Radiosity.

ELIAS, Hugo. TGLTLSBFSSP [online]. 2000 [cit. 2015-04-13]. Dostupné z:http://freespace.virgin.net/hugo.elias/radiosity/radiosity.htm

[5] Wikipedia, the free encyclopedia: Radiosity (computer graphics). In: Wikipedia: the free encyclopedia[online]. San Francisco (CA): Wikimedia Foundation, 2001- [cit. 2015-04-13]. Dostupné

z: http://en.wikipedia.org/wiki/Radiosity (computer graphics)

Authors: Ing. Rudolf Bayer, České vysoké učení v Praze, Fakulta elektrotechnická, katedra elektroenergetiky, Technická 2 , 166 27, Czech Republic, e-mail: bayerrud@fel.cvut.cz

Ing. Michal Brejcha, Ph.D., České vysoké učení v Praze, Fakulta elektrotechnická, katedra elektrotechnologie, Technická 2, 166 27, Czech Republic, e-mail: brejcmic@fel.cvut.cz 

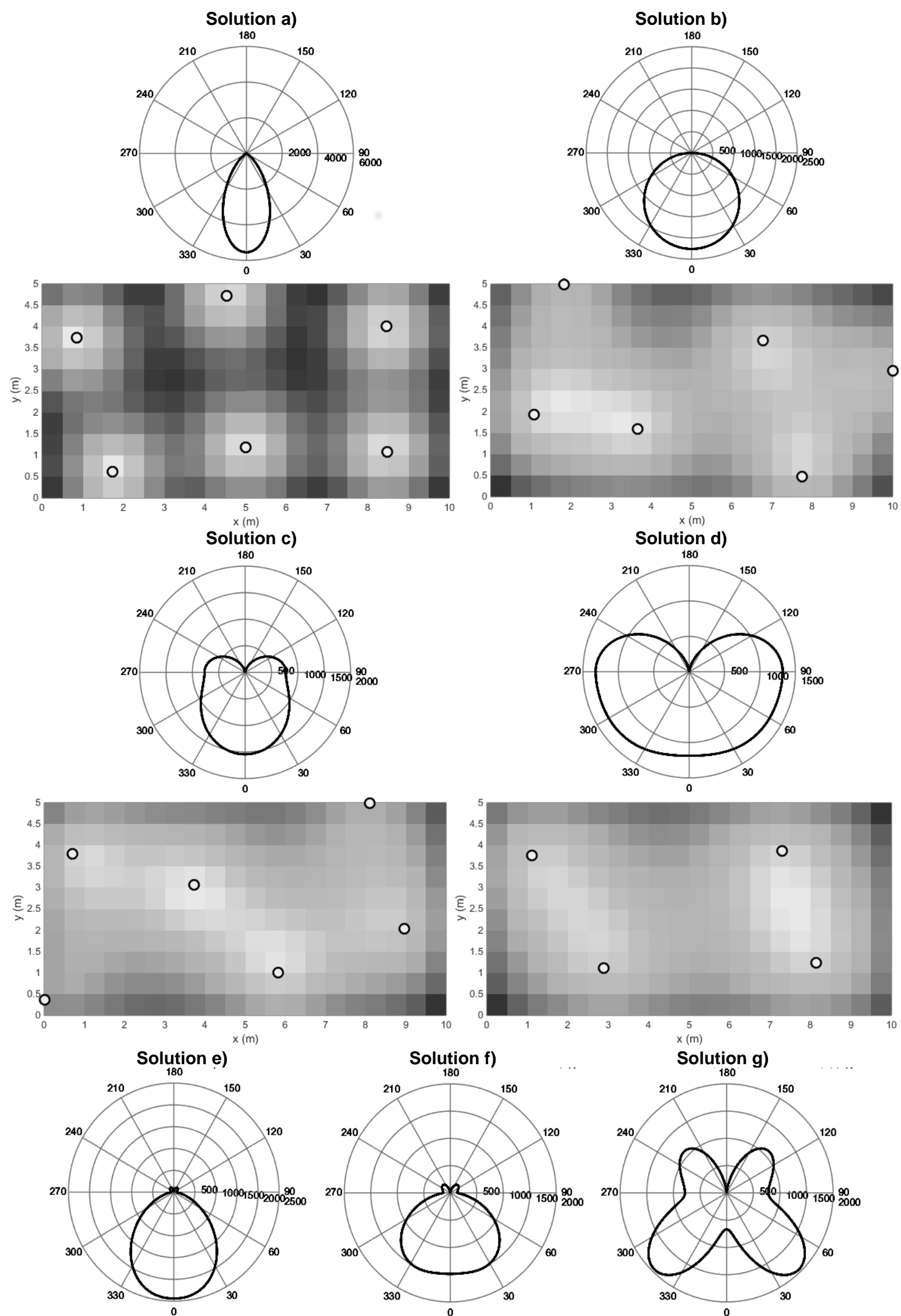

Fig. 3: List of solutions, a), b), c), d) are results of position design and e), f) and $g$ are solutions of luminuous curve design. 